\title{
Entanglement detection in optical lattices of bosonic atoms with collective measurements
}

\author{
Géza Tóth \\ Theoretical Division, Max Planck Institute for Quantum Optics, \\ Hans-Kopfermann-Straße 1, Garching, D-85748 Germany.
}

(Dated: December 5, 2018)

\begin{abstract}
The minimum requirements for entanglement detection are discussed for a spin chain in which the spins cannot be individually accessed. The methods presented detect entangled states close to a cluster state and a many-body singlet state, and seem to be viable for experimental realization in optical lattices of two-state bosonic atoms. The entanglement criteria are based on entanglement witnesses and on the uncertainty of collective observables.

PACS numbers: 03.67.-a, 03.65.Ud, 03.75.Gg, 42.50.-p, 75.10.Jm
\end{abstract}

\section{INTRODUCTION}

Recently attention has been drawn to optical lattices [1, 2, 3, 4] as promising candidates for the realization of large scale quantum information processing. Successful experiments have been done by applying state-dependent lattice potentials for atoms with two internal states. The lattices were displaced with respect to each other and then returned to their original position, making neighboring sites interact, realizing a phase gate and ultimately a spin chain dynamics [3]. These operations have recently been successfully used to entangle cold atoms on a large scale 4]. As a next step, it is very important both theoretically and for quantum information processing applications to prove that the quantum state created is entangled. This is, however, a very difficult problem [4] which has hardly been considered (with the exception of Refs. 5, 6]).

Entanglement detection in an experiment is a hard problem, since reconstructing the whole density matrix is usually not possible and the quantum state is only partially known. One can typically measure a few observables yet still would like to detect some of the entangled states. The situation is even more difficult for lattices of two-state atoms created with today's technology since the lattice sites are not accessible individually [4]. In this paper we will present scenarios where highly entangled states are detected based on very small amount of acquired knowledge: by the measurement of collective quantities. Our schemes are viable with present-day or near-future technology.

The methods to be presented detect entangled states close to cluster states [7, 8] and many-body singlets. Cluster states can easily be created in a spin chain with nearest-neighbor interaction [7] and have recently been realized experimentally in optical lattices [4]. They are more immune to decoherence than other states with genuine multi-qubit entanglement [8] and can be used as a resource for measurement based quantum computation 7]. States with total angular momentum zero are also of considerable importance. One example of such a manybody singlet state is a chain two-qubit singlets which can serve as a resource for teleportation and quantum communication. A singlet of two large spins has already been studied in a photonic system [9]. Four-qubit singlets have recently been created with photons for decoherence free quantum information processing [10]. Optical lattices arise naturally as candidates for realizing many-body singlets, for example, as a ground state of Heisenberg chains. Note that neither cluster states nor singlets are detected by the spin squeezing criterion [5], which is another approach for entanglement detection with global measurement.

All our results are based on the following simple considerations. We will build entanglement criteria with the three coordinates of the collective angular momentum, $J_{x / y / z}$. These quantities can be obtained directly by population difference measurements, without the use of multi-qubit operations. There are now two approaches for entanglement detection:

(i) Entanglement can be detected by measuring only $\left\langle J_{x / y / z}\right\rangle$ if the collective measurement is preceded by some multi-qubit quantum dynamics.

(ii) Without preceding dynamics an entanglement criterion must involve second or higher order moments of the angular momentum coordinates. However, an entangled state (e.g., a cluster state) cannot be detected this way if there exists a separable state giving the same values for the moments $\left\langle J_{x / y / z}^{m}\right\rangle$.

In this paper three necessary conditions for separability will be presented. If these are violated then the system is entangled. The first one is based on an entanglement witness, i.e. a criterion linear in expectation values 11]. Connected to it, an experimental scheme is described to measure the entanglement lifetime of a cluster state. This scheme is viable with present-day technology [4]. The second method detects also states close to a cluster state, using uncertainties of collective observables 12, 13, 14]. In both cases one has to measure a component of the collective angular momentum after an evolution under a simple Hamiltonian. The third method is based on measuring uncertainties of all the collective angular momentum components without a preceding dynamics and is a generalization of the approaches of Refs. 
[9] and [13] for detecting many-body singlets. In the following we will use the notion of spin chains and lattices of two-state atoms interchangeably.

\section{ENTANGLEMENT DETECTION WITH A WITNESS OPERATOR}

In this section we will show that for all separable states, i.e. states that can be written as

$$
\rho=\sum_{l} p_{l} \rho_{l}^{(1)} \otimes \rho_{l}^{(2)} \otimes \ldots \otimes \rho_{l}^{(N)},
$$

in a chain of $N$ qubits the following expression involving third order correlations is bounded from above:

$$
J:=\left\langle\sum_{k=1}^{N} \tilde{\sigma}_{x}^{(k)}\right\rangle \leq \frac{N}{2},
$$

where $\tilde{\sigma}_{x}^{(k)}=\sigma_{z}^{(k-1)} \sigma_{x}^{(k)} \sigma_{z}^{(k+1)}$. Here for the end of the chain $\sigma_{z}^{(0)}=\sigma_{z}^{(N+1)}=1$ and for simplicity, $N$ is taken to be even. Later it will be shown how the left hand side of Eq. (2) can be measured as the $x$ component of the collective angular momentum after an evolution under a simple Hamiltonian.

In order to prove criterion (2), first it will be proved that for a product state

$$
\begin{aligned}
J_{k} & :=\left\langle\tilde{\sigma}_{x}^{(k)}+\tilde{\sigma}_{x}^{(k+1)}\right\rangle \\
& =\left\langle\sigma_{z}^{(k-1)} \sigma_{x}^{(k)} \sigma_{z}^{(k+1)}\right\rangle+\left\langle\sigma_{z}^{(k)} \sigma_{x}^{(k+1)} \sigma_{z}^{(k+2)}\right\rangle \\
& \leq\left|\left\langle\sigma_{x}^{(k)}\right\rangle\left\langle\sigma_{z}^{(k+1)}\right\rangle\right|+\left|\left\langle\sigma_{z}^{(k)}\right\rangle\left\langle\sigma_{x}^{(k+1)}\right\rangle\right| \\
& \leq 1
\end{aligned}
$$

Note that $J_{k}$ involves a quadruplets of spins $(k-1),(k)$, $(k+1)$ and $(k+2)$. The upper bound in Eq. (3) was found using the Cauchy-Schwarz inequality and knowing that $\left\langle\sigma_{x}\right\rangle^{2}+\left\langle\sigma_{z}\right\rangle^{2} \leq 1$. This bound holds for any product state, and since $J_{k}$ is linear in expectation values, it also holds for any separable state.

The upper bound for $J$ is then $J=\sum_{k} J_{2 k+1} \leq$ $N / 2$. (Note that this sum involves $N / 2$ overlapping quadruplets corresponding to $\left.J_{1}, J_{3}, J_{5}, \ldots\right)$ This proves criterion (2). The upper bound in Eq. (2) is also the lowest possible, since the separable state $|+1\rangle_{x}|+1\rangle_{z}|+1\rangle_{x}|+1\rangle_{z} \ldots$ saturates the inequality.

Next a lower bound of the number of entangled qubit quadruplets will be deduced from the degree of violation of criterion (2). If the quadruplet of spins $(k-1),(k)$, $(k+1)$ and $(k+2)$ is separable, then $-1 \leq J_{k} \leq 1$. If it is entangled, then $-2 \leq J_{k} \leq 2$. Hence a lower limit for the number of entangled overlapping quadruplets is $J-N / 2$. The minimum number of non-overlapping entangled quadruplets is half this: $N_{q} \geq J / 2-N / 4$.

The criterion (2) is maximally violated only for a cluster state $(J=N)$. This state is defined as the eigenvector of the following three-qubit operators

$$
\sigma_{z}^{(k-1)} \sigma_{x}^{(k)} \sigma_{z}^{(k+1)}|\Psi\rangle=\lambda_{k}|\Psi\rangle,
$$

where $1 \leq k \leq N$ and $\lambda_{k} \in\{-1,+1\}$. We detect a cluster state with $\lambda_{k}=+1$ for all $k$ 's.

The spin squeezing criterion [5] does not detect cluster states as entangled. This criterion is based on the necessary condition for separability $N\left(\Delta J_{\vec{n}_{1}}\right)^{2} /\left(\left\langle J_{\vec{n}_{2}}\right\rangle^{2}+\right.$ $\left.\left\langle J_{\vec{n}_{3}}\right\rangle^{2}\right) \geq 1$, where $J_{\vec{n}_{k}}$ is the total angular momentum in the direction $\vec{n}_{k}$ and the $\vec{n}_{k}$ 's are perpendicular to each other. The state is not detected since for cluster states $\left\langle J_{\vec{n}}\right\rangle=0$ for any $\vec{n}$.

Now we will discuss how to measure $J$. It is known 7], that $U_{P G} \sigma_{x}^{(k)} U_{P G}=\sigma_{z}^{(k-1)} \sigma_{x}^{(k)} \sigma_{z}^{(k+1)}$, where $U_{P G}=$ $\exp \left\{i \frac{\pi}{4} \sum_{k}\left(1-\sigma_{z}^{(k)}\right)\left(1-\sigma_{z}^{(k+1)}\right)\right\}$ denotes an operation implementing a phase gate for all neighboring spins. Hence the three-qubit correlation terms $\tilde{\sigma}_{x}^{(k)}$ can be measured by applying $U_{P G}$ to the chain and then measuring $\sigma_{x}^{(k)}$. Also, $J$ can be obtained by applying $U_{P G}$ and then measuring the $x$ component of the collective spin. This measurement procedure can only be used to detect entanglement if the real dynamics of the system is known to sufficient accuracy [15]. (However, criterion (2) can also be used without a need for multi-qubit dynamics if the particles are individually accessible. In this case only two measurement settings are needed for the $| \pm 1\rangle_{x}| \pm 1\rangle_{z}| \pm 1\rangle_{x}| \pm 1\rangle_{z} \ldots$ and $| \pm 1\rangle_{z}| \pm 1\rangle_{x}| \pm 1\rangle_{z}| \pm 1\rangle_{x} \ldots$ bases.)

Recently, when experimentally creating a cluster state the effect of decoherence has been observed in the decreasing visibility of the interference patterns 44]. Based on the previous paragraphs, we propose the measurement of $J$ as defined in Eq. (2) to study the effect of decoherence [8] on many-body entanglement quantitatively. (Without the many-qubit dynamics it is hard to observe the decoherence of a cluster state since for the whole process $\left\langle J_{x / y / z}\right\rangle=0$.) The measurement scheme is as follows. First a cluster state is created starting from $|1111 \ldots\rangle_{x}$, followed by the application of $U_{P G}$. Then we let decoherence affect the system for time $t_{d}$. Finally, we use $U_{P G}$ again. This would ideally restore the initial state. However, due to decoherence the measurement of the collective spin $J$ in the $x$ direction will give less than the maximal $N$. The effect of decoherence can easily be followed via the decrease of $J$ with $t_{d}$.

The influence of a single phase-flip channel acting on spin $(k)$ is given by a completely positive map as $\epsilon_{k} \rho=$ $p \rho+(1-p) \sigma_{z}^{(k)} \rho \sigma_{z}^{(k)}$ where $p\left(t_{d}\right)=\left[1+\exp \left(-\kappa t_{d}\right)\right] / 2$. Assuming that all these channels act in parallel one obtains $J(p) / N=2 p-1$. (In the computations it was used that $J=N-2$ and $J=N-4$ for $\sigma_{z}^{(k)} \rho_{c l} \sigma_{z}^{(k)}$ and $\sigma_{z}^{(k)} \sigma_{z}^{(l)} \rho_{c l} \sigma_{z}^{(l)} \sigma_{z}^{(k)} ; k \neq l$, respectively.) The state of the system is detected as entangled by criterion (2) if $p>0.75$. The lower bound for the entanglement lifetime measured this way is independent of the size of the system, and as we will show, it is a quite tight bound. Following the approach of Ref. [8], one can show that the 
reduced density matrix of two neighboring qubits is entangled if $p>0.71$. The entanglement lifetime computed from criterion (2) is $20 \%$ shorter than the lifetime computed with the latter approach. The difference is slightly smaller for the partially depolarizing channel.

\section{ENTANGLEMENT DETECTION WITH UNCERTAINTY RELATIONS}

The previous approach detects cluster states with $\lambda_{k}=$ +1 eigenvalues for the defining Eqs. (4). By modifying Eq. (2), one finds that for separable states

$$
\sum_{k=1}^{N}\left\langle\tilde{\sigma}_{x}^{(k)}\right\rangle^{2} \leq \frac{N}{2} .
$$

Squaring the expectation value makes it possible to detect both $\lambda_{k}= \pm 1$. A similar approach for constructing a nonlinear expression from an entanglement witness has been recently presented in Ref. [12].

An expression equivalent to Eq. (5) can be obtained using the variances of $\tilde{\sigma}_{x}^{(k)}: \sum_{k}\left(\Delta \tilde{\sigma}_{x}^{(k)}\right)^{2} \geq N / 2$. Based on this, a collective measurement scheme can be defined with the following three operators

$$
X_{1 / 2 / 3}:=\sum_{3 k+1 / 2 / 3} \tilde{\sigma}_{x}^{(k)}
$$

$X_{1 / 2 / 3}$ is the $x$ component of the collective angular momentum operator for every third spin starting from spin $1 / 2 / 3$, after $U_{P G}$ was executed. With these, a necessary condition for separability can be obtained

$$
\sum_{m=1}^{3}\left(\Delta X_{m}\right)^{2} \geq \frac{N}{2} .
$$

The proof of Eq. (7) is as follows. For a separable state $\left(\Delta X_{1}\right)^{2}+\left(\Delta X_{2}\right)^{2}+\left(\Delta X_{3}\right)^{2} \geq \sum_{l} p_{l}\left\{\left(\Delta X_{1}\right)_{l}^{2}+\left(\Delta X_{2}\right)_{l}^{2}+\right.$ $\left.\left(\Delta X_{3}\right)_{l}^{2}\right\}=\sum_{l} p_{l} \sum_{k}\left(\Delta \tilde{\sigma}_{x}^{(k)}\right)_{l}^{2} \geq \sum_{l} p_{l}\langle N\rangle_{l} / 2=N / 2$. Here index $l$ refers to the $l$ th subensemble.

Note that while the measurement of a single operator was enough to construct the entanglement witness, an entanglement criterion with observable uncertainties usually involves at least two or three operators 12, 13, 14]. In our case, distributing the $\tilde{\sigma}_{x}^{(k)}$ terms into less then three operators would make it impossible to use $\sum_{m}\left(\Delta X_{m}\right)_{l}^{2}=\sum_{k}\left(\Delta \tilde{\sigma}_{x}^{(k)}\right)_{l}^{2}$ in the previous derivation.

\section{ENTANGLEMENT DETECTION WHEN THE PARTICLE NUMBER VARIES ON THE LATTICE}

The previous two approaches can straightforwardly be used for entanglement detection in optical lattices of bosonic atoms with two internal states, if there is a single atom per lattice site. (However, missing spins can still be easily handled with these models.) In practice, it is difficult to prepare a lattice with unit occupancy [2].

A method will now be presented which detects entangled states even if there are several atoms per lattice site, by measuring collective observables without preceding quantum dynamics. The necessary condition for separability (proved later) will be

$$
\left(\Delta J_{x}\right)^{2}+\left(\Delta J_{y}\right)^{2}+\left(\Delta J_{z}\right)^{2} \geq \frac{\langle N\rangle}{2},
$$

where $N$ is the total particle number and $J_{x / y / z}$ are the collective angular momentum coordinates. They are the sum of the corresponding single site Schwinger type angular momentum operators. For a lattice site, omitting the index $(k)$, these are defined as $j_{x}=\left(a^{\dagger} b+a b^{\dagger}\right) / 2$, $j_{y}=i\left(b^{\dagger} a-a b^{\dagger}\right) / 2$, and $j_{z}=\left(a^{\dagger} a-b^{\dagger} b\right) / 2$, where $a$ and $b$ are the bosonic destruction operators corresponding to the two internal states of the atoms. The particle number at a site is $a^{\dagger} a+b^{\dagger} b$.

If the system is in a pure state and a lattice site is not entangled with the other sites, then its state has the form $\Psi=\sum_{m} c_{m}\left|j_{m}, z_{m}\right\rangle$. A separable state is just the convex combination of products of such single site states. Here $|j, z\rangle$ is an eigenstate of $j_{x}^{2}+j_{y}^{2}+j_{z}^{2}$ with eigenvalue $j(j+1)$, and of $j_{z}$ with eigenvalue $z$. For example, $|\uparrow\rangle=$ $|1 / 2,1 / 2\rangle$ and $|\downarrow\rangle=|1 / 2,-1 / 2\rangle$ denote a single atom at the lattice site in states $a$ and $b$, respectively, while $|\emptyset\rangle=|0,0\rangle$ denotes an empty lattice site.

This representation does not take into account entanglement between particles within the lattice site, as expected, and models a lattice site as a particle with a large spin. The spin squeezing criterion [5], however, detects both entanglement between particles on the same site and entanglement between particles on different sites.

As we will show, criterion (2) is able to distinguish entanglement due to particle number variance (e.g., $|\uparrow\rangle|\emptyset\rangle+$ $|\emptyset\rangle|\uparrow\rangle)$ from entanglement in the internal states (e.g., $|\uparrow\rangle|\downarrow\rangle-|\downarrow\rangle|\uparrow\rangle)$ ). Our aim is to detect the second kind of entanglement. In the first case we have a superposition of states with different on-site particle numbers. The Schwinger operators commute with the $N_{k}$ particle number operators, thus by measuring them one cannot distinguish between a superposition and a mixture of such states [16]. Consequently an entanglement condition in terms of such observables will not take into account entanglement due to particle number variance.

The proof of criterion (8) is based on the relations

$$
\begin{gathered}
\left\langle\left(j_{x}^{(k)}\right)^{2}+\left(j_{y}^{(k)}\right)^{2}+\left(j_{z}^{(k)}\right)^{2}\right\rangle= \\
\left\langle\frac{N_{k}}{2}\left(1+\frac{N_{k}}{2}\right)\right\rangle, \\
\left\langle j_{x}^{(k)}\right\rangle^{2}+\left\langle j_{x}^{(k)}\right\rangle^{2}+\left\langle j_{x}^{(k)}\right\rangle^{2} \leq \frac{\left\langle N_{k}\right\rangle^{2}}{4} .
\end{gathered}
$$

Here Eq. (9) expresses the fact, that a two-mode bosonic system has maximal angular momentum 17]. Subtracting Eq. (10) from Eq. (9) one obtains the uncertainty 
relation for $\operatorname{spin}(k)$

$$
\left(\Delta j_{x}^{(k)}\right)^{2}+\left(\Delta j_{y}^{(k)}\right)^{2}+\left(\Delta j_{z}^{(k)}\right)^{2} \geq \frac{\left(\Delta N_{k}\right)^{2}}{4}+\frac{\left\langle N_{k}\right\rangle}{2} .
$$

For separable states $\left(\Delta J_{x}\right)^{2}+\left(\Delta J_{y}\right)^{2}+$ $\left(\Delta J_{z}\right)^{2} \geq \sum_{l} p_{l}\left\{\left(\Delta J_{x}\right)_{l}^{2}+\left(\Delta J_{y}\right)_{l}^{2}+\left(\Delta J_{z}\right)_{l}^{2}\right\}=$ $\sum_{l} p_{l} \sum_{k}\left\{\left(\Delta j_{x}^{(k)}\right)_{l}^{2}+\left(\Delta j_{y}^{(k)}\right)_{l}^{2}+\left(\Delta j_{z}^{(k)}\right)_{l}^{2}\right\}$ which together with Eq. (11) proves criterion (8). Thus the uncertainty relations (11) for the individual lattice sites gave a lower bound for the uncertainties of the corresponding collective quantities for separable states in Eq. (8) 13]. This lower bound is the highest possible, since any pure product state with unit lattice site-occupancy saturates the inequality. [For atoms on the lattice a particle number conserving superselection rule applies, thus $\left(\Delta N_{k}\right)^{2}=0$ for all pure product states.]

Inequality (8) is maximally violated for angular momentum eigenstates with total angular momentum $J=0$ (many-body spin singlet). The spin squeezing criterion 5] does not detect these states as entangled, since they have $\left\langle J_{x / y / z}\right\rangle=0$.

For two atoms at neighboring lattice sites such a singlet state is $\left|\Psi_{\text {singlet }}\right\rangle=|\uparrow\rangle|\downarrow\rangle-|\downarrow\rangle|\uparrow\rangle$. Chains of two-qubit singlets of the form $\Psi_{\text {singlet }} \otimes \Psi_{\text {singlet }} \otimes \Psi_{\text {singlet }} \otimes \ldots$ also maximally violate our necessary condition for separability (8). In general, many-body singlet states are ground states of the Hamiltonian $H=J_{x}^{2}+J_{y}^{2}+J_{z}^{2}$. Maximal violation of inequality (8) can also be obtained with the ground state of the antiferromagnetic Heisenberg chain $H=\sum j_{x}^{(k)} j_{x}^{(k+1)}+j_{y}^{(k)} j_{y}^{(k+1)}+j_{z}^{(k)} j_{z}^{(k+1)}$. With a single atom at each lattice site and for even $N$, the nondegenerate ground state is close to a superposition of chains of two-particle singlets.

It is of experimental interest that substantial violation of criterion (8) can also be achieved with a simple spin chain dynamics with ferromagnetic nearest neighbor coupling, starting out from the state $|\uparrow \uparrow \uparrow \ldots\rangle$. Finding the appropriate pulse sequence is a question of numerical optimization. For example, $U=\exp \left(-i\left\{-3.2 \sum j_{x}^{(k)} j_{x}^{(k+1)}-9.6 \sum j_{y}^{(k)} j_{y}^{(k+1)}+\right.\right.$ $\left.\left.0.8 \sum j_{z}^{(k)}\right\}\right)$ results in a $50 \%$ violation of Eq. (8) for a chain of $N=6$ atoms.

A cluster state is not detected by criterion (8) as entangled. This is not possible in general with a criterion containing only the moments $\left\langle J_{x / y / z}^{m}\right\rangle$. With straightfor- ward algebra one can prove that based on the moments for $m \leq 4(m \leq 8)$, a cluster state of $N=9(N=17)$ particles is indistinguishable from the totally mixed state $\rho_{t}^{(N)}=(|\uparrow\rangle\langle\uparrow|+| \downarrow\rangle\langle\downarrow|)^{\otimes N}$. The first non-zero moments of $\rho_{t}^{(N)}$ are $N / 4$ and $N(3 N-2) / 16$ for $m=2$ and 4 , respectively.

Beside $\left\langle J_{x / y / z}^{m}\right\rangle$, one might consider the moments of the more general angular momentum components $J_{\vec{n}}=\sum_{k=x, y, z} \alpha_{k}(\vec{n}) J_{k}$. However, the following separable state is indistinguishable from a cluster state based on any such first or second order moments: $\rho_{s}^{(N)}=\exp \left(i \pi J_{y} / 4\right)\left\{\left(|\uparrow \uparrow\rangle_{x}\langle\uparrow \uparrow|+| \downarrow \downarrow\rangle_{x}\langle\downarrow \downarrow|\right) \otimes\right.$ $\left.(|\uparrow \downarrow\rangle\langle\uparrow \downarrow|+| \downarrow \uparrow\rangle\langle\downarrow \uparrow|) \otimes \rho_{t}^{(N-2)}\right\} \exp \left(-i \pi J_{y} / 4\right)$. Here $|\uparrow\rangle_{x} /|\downarrow\rangle_{x}=|\uparrow\rangle \pm|\downarrow\rangle$. Beside having the same moments $\left\langle J_{x / y / z}^{m}\right\rangle$ for $m \leq 2$ as a cluster state, $\rho_{s}^{(N)}$ also has the same values for $A_{k l}=\left\langle J_{k} J_{l}+J_{l} J_{k}\right\rangle ; k, l=x, y, z$ $\left(A_{x y}=A_{y z}=0, A_{z x}=1\right)$.

Thus even if there exists an entanglement criterion for cluster states based on moments of the collective angular momentum components, (i) it must involve at least an angular momentum component different from $x, y$ or $z$, and (ii) it must be at least third order. This makes the detection of cluster states very difficult, if additional many-body dynamics is not used before the measurement.

\section{CONCLUSION}

In summary, we have shown how to detect entangled states close to a cluster state or a many-body spin singlet with collective measurement in an optical lattice of twostate bosonic atoms.

\section{ACKNOWLEDGMENTS}

We would like to thank H.-J. Briegel, J.I. Cirac, T. Cubitt, J.J. García-Ripoll, O. Gühne and M.M. Wolf for useful discussions. We also acknowledge the support of the European Union (Grant No. MEIF-CT-2003-500183), the EU projects RESQ and QUPRODIS, and the Kompetenznetzwerk Quanteninformationsverarbeitung der Bayerischen Staatsregierung.
[1] J.J. García-Ripoll and J.I. Cirac, New J. Phys. 5, 74 (2003); L.-M. Duan et al., Phys. Rev. Lett. 91, 090402 (2003).

[2] J.J. García-Ripoll, J.I. Cirac, Phys. Rev. Lett. 90, 127902 (2003).

[3] D. Jaksch et al., Phys. Rev. Lett. 82, 1975 (1999); G.V. Brennen et al., Phys. Rev. Lett. 82, 1060 (1999).

[4] O. Mandel et al., Nature 425, 937 (2003).

[5] A. Sørensen and K. Mølmer, Phys. Rev. Lett 83, 2274
(1999); A. Sørensen et al., Nature 409, 63 (2001).

[6] G.K. Brennen, Quant. Inf. Comp., 3, 619 (2003).

[7] H.-J. Briegel and R. Raussendorf Phys. Rev. Lett. 86, 910 (2001); R. Raussendorf and H.-J. Briegel, Phys. Rev. Lett. 86, 5188 (2001).

[8] W. Dür, H.-J. Briegel, quant-ph/0307180

[9] C. Simon and D. Bouwmeester, Phys. Rev. Lett. 91, 053601 (2003).

[10] Bourennane et al., Phys. Rev. Lett. 92, 107901 (2004). 
[11] O. Gühne et al., Phys. Rev. A 66, 062305 (2002); M. Horodecki, P. Horodecki, and R. Horodecki, Phys. Lett. A 223, 1 (1996); B. M. Terhal, Phys. Lett. A 271, 319 (2000); M. Lewenstein et al., Phys. Rev. A 62, 052310 (2000).

[12] O. Gühne, Phys. Rev. Lett. 92, 117903 (2004).

[13] H.F. Hofmann and S. Takeuchi, Phys. Rev. A 68, 032103 (2003).
[14] L.-M. Duan et al., Phys. Rev. Lett. 84, 2722 (2000); G. Tóth et al., Phys. Rev. A 68, 062310 (2003).

[15] About the precision measurement of atomic interaction parameters for optical lattices see A. Widera et. al., cond-mat/0310719

[16] F. Verstraete et al., Phys. Rev. Lett. 91, 10404 (2003).

[17] T.-L. Ho and L. Yin, Phys. Rev. Lett 84, 2302 (2000). 